\title{
Integrating candidate gene and quantitative genetic approaches to understand variation in timing of breeding in wild tit populations
}

\author{
MIRIAM LIEDVOGEL ${ }^{*}{ }^{1}$, CHARLIE K. CORNWALLIS*1 \& BEN C. SHELDON* \\ *Department of Zoology, Edward Grey Institute, University of Oxford, Oxford, UK \\ $\dagger$ Department of Biology, CAnMove Centre, University of Lund, Lund, Sweden
}

Keywords:

animal model;

breeding;

candidate gene;

circadian clock;

heritability;

polymorphism;

quantitative genetics;

timing;

wild population.

\begin{abstract}
Two commonly used techniques for estimating the effect of genes on traits in wild populations are the candidate gene approach and quantitative genetic analyses. However, whether these two approaches measure the same underlying processes remains unresolved. Here, we use these two methods to test whether they are alternative or complementary approaches to understanding genetic variation in the timing of reproduction - a key trait involved in adaptation to climate change - in wild tit populations. Our analyses of the candidate gene Clock show weak correlates with timing variables in blue tits, but no association in great tits, confirming earlier results. Quantitative genetic analyses revealed very low levels of both direct (female) and indirect (male) additive genetic variation in timing traits for both species, in contrast to previous studies on these traits, and much lower than generally assumed. Hence, neither method suggests strong genetic effects on the timing of breeding in birds, and further work should seek to assess the generality of these conclusions. We discuss how differences in the genetic control of traits, species life-history and confounding environmental variables may determine how useful integrating these two techniques is to understand the phenotypic variation in wild populations.
\end{abstract}

\section{Introduction}

Timing of reproduction is often under strong natural selection in wild populations and generally assumed to possess additive genetic variance in birds (see e.g. van der Jeugd \& McCleery, 2002; Sheldon et al., 2003; Nussey et al., 2005). Long-term studies in wild populations show that well-timed breeding, that is, timing reproduction to appropriately coincide with the peak in food abundance for their offspring, has major fitness consequences, in terms of both greater offspring survival and possibly increased parental survival (e.g. Thomas et al., 2001; Grieco et al., 2002; Sheldon et al., 2003; Visser, 2005; Charmantier et al., 2008).

Correspondence: Miriam Liedvogel, CAnMove Centre, Lund University, Sölvegatan 37, 22362 Lund, Sweden.

Tel.: +46 4622 23026; fax: +46 4622 24716;

e-mail: miriam.liedvogel@biol.lu.se

${ }^{1}$ The first two authors contributed equally to this study.
Consequently, the birds' breeding phenotype has been shaped for their ability to rear their offspring at the time of peak food abundance. To understand the evolution of the timing of breeding in natural populations, it is crucial to determine and characterize the extent to which phenotypic differences between individuals have a genetic basis (Hoekstra \& Coyne, 2007; Ellegren \& Sheldon, 2008). A number of different approaches can be used to estimate the relationship between the phenotype and the genotype. Two different commonly used approaches are as follows: (i) quantitative genetic analyses of individuals of known relatedness. This enables additive genetic variance in phenotypic traits to be measured, and hence heritability, without prior knowledge of the molecular genetic mechanisms underpinning traits (e.g. Kruuk et al., 2008; Wilson et al., 2010). (ii) In contrast, candidate gene analyses (e.g. Tabor et al., 2002; Fitzpatrick et al., 2005; Piertney \& Webster, 2010) focus on the 
association between genetic polymorphism at a targeted candidate locus (conserved across species) and phenotypic variation among individuals.

Previous research on the quantitative genetics of the timing of breeding has revealed that timing traits and cues used to adjust timing often show considerable heritability in several bird species (e.g. Merilä \& Sheldon, 2000; van der Jeugd \& McCleery, 2002; Sheldon et al., 2003; McCleery et al., 2004; Charmantier et al., 2006; Visser et al., 2011). Comparing across populations, a candidate gene approach has shown that in the variable poly-Q locus of the Clock gene, there is a latitudinal cline (Johnsen et al., 2007) with longer repeats found at higher latitudes. Birds such as blue tits show considerable variation in timing of breeding associated with latitudinal variation, and given the findings of Tauber \& Kyriacou (2005) and Johnsen et al. (2007), it is highly plausible that variation at the Clock gene underlies at least part of this variation. A within-population association of longer mean repeat lengths at the variable Clock candidate locus with later breeding in female (but not male) blue tits (Liedvogel et al., 2009) supports the hypothesized explanation that the latitudinal cline may be caused by adaptation to variation in photoperiodic parameters between populations (Johnsen et al., 2007). Therefore, there is evidence, from both quantitative genetic and candidate gene analyses, that there is genetic variation in the timing reproduction within and across populations. Candidate gene and quantitative genetic approaches have traditionally been used separately, but the extent to which these two approaches complement each other (measure different sources of genetic variation) or overlap, and whether they measure the same processes in wild populations remains unknown. However, there is potentially a lot to be gained from integrating these two techniques. First, when assessing the effect of a candidate gene on a phenotypic trait, it may allow background genetic variation to be partitioned out. This can reduce the likelihood of finding spurious correlations between candidate genes and traits (Slate et al., 2010). Second, the extent to which estimates of additive genetic variation are changed by including candidate genes in quantitative genetic analyses can help resolve whether heritability in traits arises due to many genes having small effects or few genes with large effects on the focal phenotype (Johnston et al., 2011). However, it is currently unknown whether genetic variation in the timing of reproduction estimated using candidate gene measures the same genetic variation as that estimated in quantitative genetic analyses.

Here, we combine data on a candidate gene for reproductive timing (circadian clock gene Clock), with quantitative genetic analyses to quantify the independent contributions of Clock genotype, female direct and male indirect additive genetic effects on the timing of breeding in wild populations of blue tits Cyanistes caeruleus and great tits Parus major.
The timing parameters we analyse are lay date (LD), incubation duration (ID) and observed hatch date (OH). This covers the onset of reproduction (LD), the speed of reproduction (ID) and their combined outcome in terms of when chicks hatch in the nest $(\mathrm{OH})$. We distinguish between these different parameters because they cover important aspects of reproductive scheduling and potentially allow birds to fine-tune their timing of breeding to the timing of food availability via different mechanisms. First, ID represents the interval between laying and hatching - this is often accelerated within populations when ambient temperatures are higher in order to maintain synchrony between birds and their food supply (e.g. Cresswell \& McCleery, 2003). Further, although laying date has long been the focus of studies of timing of reproduction in birds, it could be argued that laying date is only important to the extent to which it leads to the synchronization of peak food demand of nestlings with peak food supply; hatching date is a better measure of this critical date. Unfortunately, data on hatching date and ID have not always been systematically collected in field studies. We are only aware of one recent study reporting low and nonsignificant additive genetic variance and heritability estimates $\left(h^{2}=0.04 \pm 0.02, \quad V_{a}=0.073 \pm 0.036\right)$ for ID (as a female trait) in a wild population of collared flycatchers Ficedula albicollis (Husby et al., 2012). Therefore, LD is important because it determines the general timing of birds, ID is important because it is responsible for the fine-tuning of timing and hatch date is important as it is the actual match between chick demand and food supply. We prefer to report analyses of all three traits while acknowledging their interdependence: all are relevant in understanding the seasonality of timing in birds.

We first fitted models to test for the effect of the candidate gene Clock on variation in timing variables. Second, we quantify additive genetic variance in timing traits using a quantitative genetic model (animal model). Quantitative genetic analyses have often treated the timing of reproduction as a female-limited trait. However, it has recently been suggested that males may indirectly influence female laying dates (e.g. improving her pre-reproductive condition by food provisioning), and thus, a more realistic way to model the timing of reproduction is to estimate both a direct female additive genetic effect and an indirect genetic effect of the pair male (Brommer \& Rattiste, 2008). We therefore use animal models to estimate female direct and male indirect genetic effects on the timing of reproduction. Finally, we combined both approaches in an integrated model to test whether the two methods estimate similar effects. Models including both candidate gene and additive genetic effects allowed us to quantify the extent to which variation in the number of poly-Q clock repeats explains estimates of quantitative genetic variation in the timing of reproduction. 


\section{Methods}

\section{Blue tit data}

All data were collected as a part of an ongoing long-term study (data collected between 2001 and 2008 included here in the full blue tit data set) on the breeding ecology of blue tits in Wytham Woods, UK, as previously described (Liedvogel et al., 2009). In short, the breeding population was monitored from nest-building until fledging of young. Parents and offspring were caught, identified and individually marked. Standard biometric measurements and blood samples were collected under licence. Only birds with exact information for all of the following phenotypic traits were included in our analyses: lay date $(\mathrm{LD}$, that is, day when the female lays the first egg, only first clutches are included), $\mathrm{OH}$ and clutch size (CS). These data allow precise calculation of ID, defined as $\mathrm{OH}-(\mathrm{LD}+\mathrm{CS})$ for each breeding event.

In total, our full data set comprises 3090 individual blue tits and a total of 4024 breeding events between 2001 and 2008. This includes 2361 birds with a single breeding attempt, 562 birds breeding twice, 136 birds with three, 24 birds with four and seven birds with five breeding attempts, respectively (Table 1 : full blue tit data set). Of the 3090 individuals, we had data on the Clock genotype of 851 individuals representing 1158 breeding events, with 638 birds breeding once, 137 birds breeding twice, 55 birds with three, 14 with four and five individuals with five breeding events, respectively (Table 1: clock data set blue tits).

Birds were genotyped at the Clock locus by extracting genomic DNA, and the length Clock polymorphism was measured for all breeders in 2006 and 2007 as previously described (Liedvogel et al., 2009). In summary, the variable glutamine-rich locus of the Clock gene was characterized by PCR amplification using a previously described primer set (Johnsen et al., 2007). The Clock genotype (i.e. number of glutamine repeats) was determined using an ABI PRISM genetic analyzer 3100, and allele sizes were resolved by GeneMapper 3.7 (for details, see Johnsen et al., 2007; Liedvogel et al., 2009).

A social pedigree was constructed based on identifying parents from unique ring numbers when feeding young (both sexes) or in the late stages of incubation (females only). Nestlings were ringed before fledging enabling connection of parents with offspring. The entire blue tit pedigree contained 23023 individuals, of which all were informative for quantitative genetic analyses of the full phenotypic data set. This represented 19713 maternities, 19664 paternities, 96287 full-siblings and 169110 halfsibling relationships. From the whole pedigree, 904 individuals were informative for the genetic relationships between individuals in the clock data set that included 164 maternities, 159 paternities and 43 full-siblings and 74 half-siblings (Figure S1). Pedigree statistics and supplementary figures were produced using pedantics R package (Morrissey \& Wilson, 2010).

\section{Great tit data}

Great tits have been studied in Wytham woods since 1947, which encompasses the blue tit study period (Perrins, 1965; McCleery et al., 2004; Charmantier et al., 2008). Phenotypic data collection, DNA extraction and Clock genotyping procedures were carried out in the same way as for blue tits (for details, also see Liedvogel $\&$ Sheldon, 2010). Our full data set for great tits comprises 12724 individuals and a total of 19395 breeding events between 1947 and 2008. The number of monitored breeding events per individual ranged from one to eight (one bird), with an average of 1.5 breeding attempts per individual. The data set of great tits with known Clock genotypes comprised 572 individuals with a total of 1028 breeding events.

The complete great tit pedigree contained 156305 individuals, of which all were informative for the analysis of the full data set and included 73838 maternities, 73846 paternities, 314558 full-siblings and 707538 half-sibling relationships. From the whole pedigree, 1548 were informative for genetic relationships between individuals in the clock data set. This included 694 maternities, 661 paternities, 154 full-siblings and 325 half-siblings (Figure S2).

\section{Blue tit statistical analyses}

For each timing variable (lay date, incubation duration and observed hatch), we ran a series of three analyses:

Table 1 Differences in pedigree structure of blue tit and great tit full data set and data subsets restricted to individuals with known Clock genotype (clock data set). Data for maternal and paternal sibship sizes are presented as mean $( \pm \mathrm{SE})$.

\begin{tabular}{|c|c|c|c|c|c|}
\hline Data set & $n_{\text {dams }}$ & Maternal sibship size & $n_{\text {sires }}$ & Paternal sibship size & $\begin{array}{l}\text { Mean pairwise } \\
\text { relatedness }\end{array}$ \\
\hline Blue tit full data set & 1958 & $10.07 \pm 0.15$ & 2062 & $9.54 \pm 0.15$ & 0.0005 \\
\hline Blue tit clock data set & 115 & $1.43 \pm 0.06$ & 108 & $1.47 \pm 0.06$ & 0.0006 \\
\hline Great tit full data set & 6790 & $10.87 \pm 0.09$ & 7485 & $9.87 \pm 0.07$ & * \\
\hline Great tit clock data set & 497 & $1.40 \pm 0.03$ & 479 & $1.38 \pm 0.03$ & 0.002 \\
\hline
\end{tabular}

*Calculation of pairwise relatedness for the size of the full great tit pedigree was not possible with the available computer power, but numbers for full- and half-sibs in combination with sibship sizes should give an adequate estimate of pedigree structure. 
first, we analysed the variation in timing variables explained by the candidate gene Clock (PQ) using a linear mixed effects model (LMM) with restricted maximumlikelihood estimation (REML) entering the number of poly-Q repeats as a fixed effect (covariate). In addition, we fitted fixed effects to control for age (2-level factor: 1st year, >1 year old) and sex (2-level factor). Wytham woods is a heterogeneous woodland, divided into nine sectors showing different habitat characteristics (Minot $\delta$ Perrins, 1986). The study site also encompasses $>100 \mathrm{~m}$ variation in altitude, which is sufficient to cause variation in the phenology of plants and insects owing to differences in mean temperature. Altitude has consistently been shown to be correlated with variation in the timing of breeding between individuals within years (e.g. Wilkin et al., 2006). We controlled for this topographical variation by entering the altitude of each breeding attempt as a fixed effect (covariate), which was calculated using available data originally extracted from an inverse distance weighting (IDW) interpolation of a 50-m resolution Land Form Digital-Terrain-Model (DTM) data set provided by Ordnance Survey (from Wilkin et al., 2006). We also entered breeding density as a fixed effect (covariate), which was calculated per sector per year as this is known the effect reproductive timing (see Wilkin et al., 2006). We also included year, nest box, female identity and male identity as random effects, to account for yearly variation, repeated breeding attempts in the same nest box and repeated breeding attempts by individual females and males (permanent environment effects: pe), respectively (model 1).

Second, we used an 'animal model' with REML that uses the pedigree to specify a relationship (co)variance matrix between individuals that enables additive genetic variation in traits to be quantified (Lynch \& Walsh, 1998). This allowed total variation in timing traits ( $V_{P}=$ sum of variance components) to be partitioned into a direct female genetic effect $\left(V_{(a f)}\right)$, a male indirect genetic effect $\left(V_{(a m)}\right)$, different components of environmental variation (random effects: year, nest box, female pe, male pe) and residual variation $\left(V_{e}\right)$ while estimating the same fixed effects (age, sex, breeding density, altitude) as in the analysis of Clock gene effects $(P Q)$. Therefore, we used exactly the same model as in the first analyses, but removed the fixed effect of $P Q$ and fitted random effects to estimate female direct and male indirect genetic effects (model 2). Variance components were constrained to be positive, and therefore, the heritability of female direct genetic effects and male indirect genetic effects was calculated as $h_{f}^{2}=V_{(a f)} / V_{P}$ and $h_{m}^{2}=V_{(a m)} / V_{P}$, respectively. Standard errors of heritability estimates were calculated using the 'delta' method as suggested by Venables \& Ripley (2000), S programming p 170 using $\mathrm{R}$ code written by Ian White. Finally, we integrated the approaches used in the first and second analyses to quantify the independent contributions of Clock genotype $(P Q), \quad V_{(a f)}$ and $V_{(a m)}$ to variation in the timing of reproduction variables. This was carried out by entering $P Q$ as a fixed effect to the model used for assessing $V_{(a f)}$ and $V_{(a m)}$ (model 3). If genetic variation measured by $P Q$ overlaps with $V_{(a f)}$ and $V_{(a m)}$, then the parameter estimates of $P Q$ in model 3 will be different from model 1 and the estimates of $V_{(a f)}$ and $V_{(a m)}$ in model 2 and 3 will be different. However, model 3 was only performed if estimates of direct and indirect genetic effects in model 2 and the parameter estimate of PQ on timing traits in model 1 were $>0$. In other words, if there was a lack of additive genetic variation or the Clock gene did not explain any variation in any of the timing traits, then model 3 was not conducted.

We modelled the timing of reproduction as:

$$
\begin{gathered}
y_{i} \sim \mu+P Q+\text { age }+ \text { breeding density }+ \text { altitude } \\
\quad+\text { year }+ \text { nest box }+p e_{f}+p e_{m}+e \quad(\text { model } 1) \\
y_{i} \sim \mu \\
+ \text { age }+ \text { breeding density }+ \text { altitude }+ \text { year } \\
\quad+\text { nest box }+p e_{f}+p e_{m}+a_{f}+a_{m}+e \quad(\text { model } 2) \\
y_{i} \sim \mu+P Q+\text { age }+ \text { breeding density }+ \text { altitude }+ \text { year } \\
\quad+\text { nest box }+p e_{f}+p e_{m}+a_{f}+a_{m}+e \quad(\text { model } 3)
\end{gathered}
$$

where $p e_{f}$ and $p e_{m}$ are female and male permanent environment effects and $a_{f}$ and $a_{m}$ are direct female and indirect male genetic effects, respectively, and $e$ is residual variation. $P Q$ was first treated as a fixed effect because we were interested in testing the directional effects of number of poly-Q repeats on the mean timing of reproduction. However, we also calculated the \% of $V_{P}$ explained by $P Q$, and $V_{(a f)}$ and $V_{(a m)}$ by entering $P Q$ as a random effect (7 levels), which gave qualitatively and quantitatively similar results (Tables S27-32).

\section{Great tit statistical analyses}

We conducted exactly the same analyses on great tits as we did for blue tits. However, there were two differences due to the way data were collected: First, age was treated as a continuous variable rather than categorical (juvenile vs adult) as the great tit population has been monitored since 1947 as opposed to 2001 for blue tits. Therefore, it was possible to enter the exact age of individuals into models. Second, of the 606 great tits genotyped, only 34 were males and therefore analyses on the clock data set were restricted to only females.

\section{Analyses to assess the ability to detect heritability using the clock data sets}

We performed two sets of analyses to assess whether it was possible to detect additive genetic variance using the clock data set and associated pedigree.

First, we analysed data on the timing variables using the full data set expanding our sample size for blue tits from 482 females and 369 males to 1687 females and 
1398 males, and our great tit sample size from 572 females to 6540 females and 5366 males (full great tit data set). This allowed us to compare the levels of additive genetic variance estimated with the clock data set to much larger data sets that have sample sizes that exceed previously published studies with significant estimates of heritability (see Table 1 for differences in pedigree structure of the full and restricted data sets).

Second, we took a trait, fledging mass, that we knew had high additive genetic variance and heritability and examined how estimates of genetic variation changed when we used our full data set and the clock data set (Garant et al., 2004; Tables S14 \& S26). In order to replicate the same data structure for fledging mass as for timing traits, we randomly selected the fledging mass of a single chick so that there was only one phenotypic value per set of parent (See Table S28 for details). We then calculated additive genetic variance and heritability in fledging mass using the full data set and the data set that only included birds that had been Clock genotyped. If the clock data set and associated pedigree were not sufficient to recover estimates of additive genetic variance and heritability, then we expected that (i) analyses of timing traits using the full data set would yield higher estimates of additive genetic variance and heritability than the clock data set and (ii) significant estimates of additive genetic variance and heritability in fledging mass gained using the full data set would disappear when analysing the clock data set.

In all analyses, covariates were Z-transformed prior to analyses. The significance of fixed effects was examined using conditional Wald $F$ statistics (Gilmour et al., 2009). Nonsignificant interactions were sequentially removed from models starting with the term with the highest $P$ value until only main effects and significant interactions remained. The significance of random effects was assessed using log-likelihood ratio tests (LRTs) (Self \& Liang, 1987). Parameter estimates are presented with standard errors (SE) unless stated otherwise. All analyses were conducted in ASReml-R version 3 (Gilmour et al., 2009).

\section{Results}

\section{Blue tit candidate gene analyses}

Candidate gene analyses confirm the general pattern found in our earlier study (Liedvogel et al., 2009). After controlling for the effects of breeding density and altitude (Table S1-S9, i.e. birds generally breed earlier at lower altitudes and in areas of high density), ID was significantly correlated with fewer poly-Q repeats at the variable Clock locus $\left(0.08 \pm 0.04, F_{1,860}=4.16, P=0.04\right.$; Table 2 \& S4). In line with previous results, blue tits that laid and hatched chicks earlier had shorter Clock alleles, but these results were nonsignificant $\left(\mathrm{LD}: F_{1,889}=0.52\right.$, $P=0.47$; OH: $F_{1,885}=0.10, P=0.75$. Tables $\left.2, \mathrm{~S} 1, \mathrm{~S} 7\right)$. There was no evidence for a sex by genotype interaction in any of the timing traits (all results with $P>0.41$; Tables S1, S4, S7). There were highly significant annual variation and large differences between breeding nest boxes in all timing traits $(P<0.0001$ for year and nest

Table 2 Estimating the relative contributions of the Clock gene (quantified as number of poly-Q repeats, $P Q$ ), female direct genetic effects $\left(V_{a f}\right)$ and male indirect genetic effects $\left(V_{a m}\right)$ to variation in the timing of reproduction in blue tits.

\begin{tabular}{|c|c|c|c|c|c|c|c|c|c|}
\hline Traits & Analysis & $P Q$ & $V_{o b s}$ & $V_{a f}$ & $h_{f}^{2}$ & $V_{a m}$ & $h_{m}^{2}$ & $V_{a}$ & $h^{2}$ \\
\hline \multirow[t]{4}{*}{ Lay date } & $C G$ & $-0.09 \pm 0.12(0.47)$ & 43.37 & - & - & - & - & - & - \\
\hline & QG & - & & $1.08 \pm 0.84(0.41)$ & $0.02 \pm 0.02$ & $0.00 \pm 0.00(1.00)$ & $0.00 \pm 0.00$ & - & - \\
\hline & $C G \& Q G$ & $-0.09 \pm 0.12(0.45)$ & & $1.10 \pm 0.85(0.39)$ & $0.02 \pm 0.02$ & $0.00 \pm 0.00(1.00)$ & $0.00 \pm 0.00$ & - & - \\
\hline & Full QG & - & 54.07 & $1.41 \pm 2.08(0.52)$ & $0.02 \pm 0.04$ & $0.69 \pm 0.73(0.40)$ & $0.01 \pm 0.01$ & - & - \\
\hline \multirow[t]{4}{*}{ Incubation } & $C G$ & $0.08 \pm 0.04(0.04)$ & 3.10 & - & - & - & - & - & - \\
\hline & $Q G$ & - & & $0.01 \pm 0.09(0.86)$ & $0.00 \pm 0.00$ & $0.00 \pm 0.00(1.00)$ & $0.00 \pm 0.00$ & - & - \\
\hline & $C G \& Q G$ & $0.08 \pm 0.04(0.04)$ & & $0.00 \pm 0.00(1.00)$ & $0.00 \pm 0.00$ & $0.00 \pm 0.00(1.00)$ & $0.00 \pm 0.00$ & - & - \\
\hline & Full QG & - & 4.03 & $0.00 \pm 0.00(1.00)$ & $0.00 \pm 0.00$ & $0.00 \pm 0.00(1.00)$ & $0.00 \pm 0.00$ & - & - \\
\hline \multirow[t]{4}{*}{ Hatch date } & $C G$ & $-0.03 \pm 0.10(0.75)$ & 43.01 & & & & & - & - \\
\hline & $Q G$ & - & & $0.60 \pm 0.57(0.39)$ & $0.01 \pm 0.01$ & $0.00 \pm 0.00(1.00)$ & $0.00 \pm 0.00$ & - & - \\
\hline & $C G \& Q G$ & $-0.04 \pm 0.10(0.72)$ & & $0.61 \pm 0.57(0.38)$ & $0.01 \pm 0.01$ & $0.00 \pm 0.00(1.00)$ & $0.00 \pm 0.00$ & - & - \\
\hline & Full QG & - & 46.31 & $2.78 \pm 1.41(0.06)$ & $0.05 \pm 0.03$ & $0.59 \pm 0.50(0.37)$ & $0.01 \pm 0.01$ & - & - \\
\hline \multirow{2}{*}{$\begin{array}{c}\text { Fledging } \\
\text { mass }\end{array}$} & $Q G$ & - & 1.06 & - & - & - & - & $0.56 \pm 0.11(<0.0001)$ & $0.46 \pm 0.09$ \\
\hline & Full QG & - & 2.25 & - & - & - & - & $0.65 \pm 0.05(<0.0001)$ & $0.27 \pm 0.02$ \\
\hline
\end{tabular}

Data presented are the parameter estimates $( \pm \mathrm{SE})$ of the relationship between timing variables and $P Q$, and the variance components and heritabilities $( \pm \mathrm{SE})$ of $V_{a f}$ and $V_{a m}$ in timing variables estimated using LMMs from the different analyses.

$\mathrm{CG}=$ candidate gene analyses on clock data set, $\mathrm{QG}=$ quantitative genetic analysis on clock data set, $\mathrm{CD} \& \mathrm{QG}=$ combined candidate gene and quantitative analysis on clock data set, Full QG = quantitative genetic analysis on full data set.

Estimates of $V_{a}$ in fledging mass are given for comparison.

$V_{\text {obs }}$ is the raw phenotypic variance.

$P$ values are presented in parenthesis. 
box in all traits; Tables S1, S4, S7). However, permanent environment effects due to females were small and nonsignificant and undetectable in males (Tables S1, S4, S7).

\section{Quantitative genetic analyses}

The magnitude of quantitative genetic parameters and their associated heritabilities in blue tits was extremely small for all timing traits and much lower than expected. This was true for analyses of both the full data set and the restricted clock data set. Estimates of female direct genetic effects calculated using the clock data set were very low with heritabilities of $0.02 \pm 0.02$ (LD), 0 (ID) and $0.01 \pm 0.01(\mathrm{OH})$ (Tables 2, S2, S5, S8). The results were very similar when analysing the full data set with heritabilities estimated as $0.02 \pm 0.04$ (LD), 0 (ID) and $0.05 \pm 0.03$ (Tables 2, S10-S12). Male indirect genetic effects were not detectable in any of the analyses (Table 2). Effects of other fixed (breeding density, altitude) and random (year, nest box) effects were very similar to those in the candidate gene analyses (Tables S1-S9).

\section{Integrating candidate gene and quantitative genetic analyses}

Estimates of Clock gene effects that tested the contribution of both Clock genotype and quantitative genetic effects (model 3: Tables 2, S3, S6, S9) were almost identical compared to those that only included candidate gene effects (model 1). Similarly, estimates of quantitative genetic effects were unaffected by entering Clock genotype into models (Tables 2, S3, S6, S9). We found very similar results entering the effect of Clock as a random effect with estimates of female direct genetic effects and male indirect genetic effects being unaffected by the inclusion of Clock (Tables S27-S29). The low estimates of genetic variation in timing traits were not due to the structure of our data set as similar estimates were found using the full data set and estimates of additive genetic variance and heritability in fledging mass were comparable across the full data set and the clock data set (Tables S13-S14). This indicates that both the full and restricted clock data sets used for our analyses are sufficiently large to estimate additive genetic variance and calculate trait heritability. Together this suggests that timing of reproduction is largely environmentally determined in this blue tit population.

\section{Great tit candidate gene and quantitative genetic analyses}

To investigate the generality of our findings for blue tits, we carried out the same analyses on great tits, a related species inhabiting the same woodlands. Overall, the results for both species were very similar. In line with previous analyses, we find no effect of Clock genotype on LD, ID or hatch date when Clock was entered as a fixed effect (all results with $P>0.62$; Tables 3, S17) or as a random effect (Tables S30-S32). Similarly to blue tits, we found highly significant annual variation in all timing traits $(P<0.0001$ for year in all timing traits; Table S17). Variation in LD and $\mathrm{OH}$ explained by differences between breeding nest boxes showed a similar, although weaker pattern, than in blue tits (variance components \pm SE: $\mathrm{LD}=3.05 \pm 1.49, \quad \mathrm{LRT}=5.13, \quad P=0.02 . \quad \mathrm{OH}=2.13 \pm$ 1.21, LRT $=3.51, P=0.06)$, but did not explain the variation in ID $(0.05 \pm 0.15, \mathrm{LRT}=0.13, P=0.72)$.

Table 3 Estimating the relative contributions of the Clock gene $(P Q)$, female direct genetic effects $\left(V_{a f}\right)$ and male indirect genetic effects $\left(V_{a m}\right)$ to variation in the timing of reproduction in great tits.

\begin{tabular}{|c|c|c|c|c|c|c|c|c|c|}
\hline Trait & Analysis & $P Q$ & $V_{o b s}$ & $V_{a f}$ & $h_{f}^{2}$ & $V_{a m}$ & $h_{m}^{2}$ & $V_{a}$ & $h^{2}$ \\
\hline \multirow[t]{3}{*}{ Lay date } & CG & $-0.11 \pm 0.12(0.62)$ & 50.77 & - & - & - & - & - & - \\
\hline & QG & - & & $0.00 \pm 0.00(1.00)$ & $0.00 \pm 0.00$ & - & - & - & - \\
\hline & Full QG & - & 83.03 & $2.62 \pm 0.67(<0.0001)$ & $0.03 \pm 0.01$ & $0.75 \pm 0.41(0.16)$ & $0.01 \pm 0.01$ & - & - \\
\hline \multirow[t]{4}{*}{ Incubation } & CG & $-0.02 \pm 0.07(0.73)$ & 3.97 & - & - & - & - & - & - \\
\hline & QG & - & & $0.62 \pm 1.08(0.63)$ & $0.15 \pm 0.27$ & - & - & - & - \\
\hline & $C G \& Q G$ & $-0.02 \pm 0.07(0.74)$ & & $0.62 \pm 1.08(0.63)$ & $0.15 \pm 0.27$ & - & - & - & - \\
\hline & Full QG & - & 4.40 & $0.00 \pm 0.00(1.00)$ & $0.00 \pm 0.00$ & $0.00 \pm 0.00(1.00)$ & $0.00 \pm 0.00$ & - & - \\
\hline \multirow{3}{*}{$\begin{array}{l}\text { Hatch } \\
\text { date }\end{array}$} & CG & $-0.05 \pm 0.20(0.80)$ & 44.69 & & & - & - & - & - \\
\hline & QG & - & & $0.00 \pm 0.00(1.00)$ & $0.00 \pm 0.00$ & - & - & - & - \\
\hline & Full QG & - & 70.72 & $1.57 \pm 0.52(0.0003)$ & $0.02 \pm 0.01$ & $0.48 \pm 0.31(0.17)$ & $0.01 \pm 0.01$ & - & - \\
\hline \multirow{2}{*}{$\begin{array}{l}\text { Fledging } \\
\text { mass }\end{array}$} & QG & - & 3.10 & - & - & - & - & $1.34 \pm 0.22(<0.0001)$ & $0.40 \pm 0.07$ \\
\hline & Full QG & - & 2.40 & - & - & - & - & $1.30 \pm 0.02(<0.0001)$ & $0.51 \pm 0.01$ \\
\hline
\end{tabular}

Data presented are the parameter estimates $( \pm \mathrm{SE})$ of the relationship between timing variables and $P Q$, and the variance components and heritabilities $\left( \pm \mathrm{SE}\right.$ ) of $V_{a f}$ and $V_{a m}$ in timing variables estimated using LMMs from the different analyses (CG = candidate gene analyses on clock data set, QG = quantitative genetic analysis on clock data set, CD \& QG = combined candidate gene and quantitative analysis on clock data set, Full QG = quantitative genetic analysis on full data set). Estimates of $V_{a}$ in fledging mass are given for comparison. $V_{\text {obs }}$ is the raw phenotypic variance. $P$ values are presented in parenthesis 
Quantitative genetic analyses of the great tit clock data set revealed that, as with blue tits, very little variation in LD, ID and $\mathrm{OH}$ was explained by direct female genetic effects (Tables 3, S1 5, S17, S20). When analysing the full data set, we found small, but significant, female direct genetic variance in LD and hatch date, but not ID (LD: $2.62 \pm 0.67$, $\mathrm{LRT}=22.61, P<0.0001 ; \mathrm{OH}: 1.57 \pm 0.52, \mathrm{LRT}=12.80$, $P=0.0003$ ), with low heritabilities of $0.03 \pm 0.01$ (LD), 0 (ID) and $0.02 \pm 0.01(\mathrm{OH})$ (Tables 3, S22-S24). As was the case with blue tits, estimates of the Clock gene and quantitative genetic parameters were unaffected by each other; models including both effects (model 3) yielded the same estimates as models including only separate effects (model 1 \& 2) (Tables 3, S16, S19, S21).

\section{Discussion}

In this study, we used candidate gene and quantitative genetic approaches to understand the genetic basis to variation in timing of reproduction in wild tit populations. In line with previous research, we detected a weak but consistent effect of Clock genotype on breeding phenotype in blue tits with fewer poly-Q repeats at the variable candidate locus being significantly associated with shorter incubation times. This differed markedly from great tits where there was no variation in allele frequency at the candidate locus precluding any relationship with the timing of breeding. Our quantitative genetic analyses show that, in contrast to previous studies, heritability estimates for timing traits in both blue tits and great tits were much lower than previous studies of these traits. We found very little direct female or indirect male additive genetic effects on the timing of reproduction in either species. Consequently, we found little overlap in the genetic variation estimated by the candidate gene Clock and quantitative genetic analyses in these two study populations. However, it is important to highlight that this may simply be due to very low genetic variation in timing variables per se, restricting our ability to test the original idea that quantitative genetic estimates may be changed by accounting for variation in candidate genes. Together our results suggest that timing of reproduction, at least in the populations we investigated, are largely environmentally determined and poor targets for assessing the relative merits of these two genetic approaches, contrary to the original hypotheses framed at the beginning of this work.

Previous research in a number of populations has returned reasonable estimates of additive genetic variance for timing of breeding in birds (e.g. Merilä $\delta$ Sheldon, 2000; van der Jeugd \& McCleery, 2002; Sheldon et al., 2003; McCleery et al., 2004), raising the question as to why our estimates differ from previous work (summarized in Table 4)? We suggest that there are two main reasons for these differences: first, different methodological approaches ('animal model' versus parent-offspring regression, see Table 4) will inevitably lead to differences in heritability estimates, as more sophisticated models and larger data sets allow a better identification and separation of genetic and environmental sources of variation in target traits. Second, in the analyses here, we separated confounding sources of variation such as nest box location, year of reproduction, age, breeding density, altitude and male and female specific effects on timing. Wilson (2008) pointed out that heritability estimates from mixed models $\left(V_{a} / V_{a}+V_{e}\right.$ in the simplest form) are highly dependent upon the fixed effects entered into models because it changes the magnitude of residual variation $\left(V_{e}\right)$ and estimates of $V_{a}$ can be reduced when fixed effects are genetically correlated with response traits. In previous analyses, these effects have not always been accounted for, and if genotypes vary (or covary) systematically with any of these variables this can lead to elevated estimates of heritability (e.g. van der Jeugd \& McCleery, 2002). We found in great tits that if we excluded all fixed and random factors apart from male and female permanent environment effects and female direct and male indirect genetic effects, we were able to recover similar estimates of genetic variance in LD to previous studies $\left(h^{2}\right.$ for female direct effect $=0.17, h^{2}$ for indirect male effect $=0.16$, Table S36). In contrast, removing fixed and random effects from models of LD in blue tits had very little effect on estimates of direct female and indirect male genetic effects (Tables S33-38). This highlights that the environmental contexts where timing of reproduction is heritable across different species require further investigation.

Importantly, heritabilities are not necessarily constant, and thus estimates using the same models and the same species may reveal different results. Different heritability estimates can be due to changes in genetic variance (e.g. changes in allele frequency due to selection, migration, inbreeding), changes in phenotypic variation due to different environmental condition, or the correlation between genes and environment can change. For example, higher spring temperature may have led to stronger selection for earlier breeding reducing $V_{a}$. It is worth noting that the data we used on the timing of reproduction were the most recent of all studies in Table 4 and included some of the earliest breeding attempts in the 65 -year period that the great tit population has been monitored. Changes in $V_{a}$ may also be explained by variation in dispersal, that is, higher dispersal resulting in higher environmental variation and thus selection for phenotypic plasticity rather than just early breeding (e.g. Nussey et al., 2005; Charmantier et al., 2008).

Dispersal rate and distance also influence the ability to separate permanent environment effects from additive genetic variance. In our case, average dispersal distance for great tits is smaller than for blue tits (e.g. Matthysen et al., 2005), and we thus expect that lower dispersal ultimately leads to a greater chance of being re-sighted and thus the level of accuracy in estimating permanent 
Table 4 Summary of heritability estimates $h^{2}$ and variance components (confidence intervals) for timing of breeding traits ( $L D$ laying date, $O H$ observed hatch, $I D$ incubation duration) in different bird species/populations. Methodological approaches used to estimate heritability are (A) quantitative genetic models (animal model) and $(B)$ parent-offspring regressions.

\begin{tabular}{|c|c|c|c|c|c|c|c|}
\hline A species (population) & Trait & $n$ & $V_{a}$ & $h_{f}^{2}$ & $h_{m}^{2}$ & Effects included & Study \\
\hline BT (Wytham, UK) & LD & $\begin{array}{l}\text { (f) } 1687 \\
\text { (m) } 1398 \\
\text { (ba) } 4024\end{array}$ & $\begin{array}{l}\text { (f) } 1.41(2.08) \\
\text { (m) } 0.69(0.73)\end{array}$ & $0.02(0.04)$ & $0.01(0.01)$ & $\begin{array}{l}\text { Age, sex, breeding density, altitude } \\
(\mathbf{F}) \text { year, nest box, } p e_{f}, p e_{m}(\mathbf{R})\end{array}$ & [1] \\
\hline GT (Wytham, UK) & LD & $\begin{array}{l}\text { (f) } 6540 \\
\text { (m) } 5366 \\
\text { (ba) } 9487\end{array}$ & $\begin{array}{l}\text { (f) } 2.62(0.67) \\
\text { (m) } 0.75(0.41)\end{array}$ & $0.03(0.01)$ & $0.01(0.01)$ & $\begin{array}{l}\text { Age, sex, breeding density, altitude }(\mathbf{F}) \\
\text { year, nest box, } p e_{f}, p e_{m}(\mathbf{R})\end{array}$ & \\
\hline BT (Muro, Corse, FR) & LD & (ba) 454 & (f) $19.4(4.0)$ & $0.43(0.07)$ & & Age, year (F) & [2] \\
\hline BT (Pirio, Corse, FR) & LD & (ba) 1228 & (f) $5.2(3.2)$ & $0.20(0.12)$ & & Age, year $(\mathbf{F})$ & \\
\hline GT (Hoge Veluwe, NL) & LD & (ba) 2469 & 4.26 & $0.17(0.03)$ & & $\begin{array}{l}\text { Year, age (F) } \\
f \mid \mathrm{D}(\mathbf{R})\end{array}$ & [3] \\
\hline $\begin{array}{l}\text { GT (Wytham, UK) } \\
(1965-1988)\end{array}$ & LD & $\begin{array}{l}\text { (f) } 2450 \\
\text { (ba) } 3575\end{array}$ & $7.57(1.48)$ & $0.16(0.03)$ & & $\begin{array}{l}\text { Age }(\mathbf{F}) \\
\text { year, ID (R) }\end{array}$ & [4] \\
\hline $\begin{array}{l}\text { GT (Wytham, UK) } \\
\text { (1989-2004) }\end{array}$ & LD & $\begin{array}{l}\text { (f) } 2285 \\
\text { (ba) } 3357\end{array}$ & $5.43(1.36)$ & $0.09(0.03)$ & & Age $(\mathbf{F})$ year, ID $(\mathbf{R})$ & \\
\hline GT (Wytham, UK) & LD & (f) 1777 & $5.02(1.879)$ & $0.16(0.06)$ & & Age, habitat (cohorts) $(\mathbf{F})$ nest box $(\mathbf{R})$ & {$[5]$} \\
\hline CF (Gotland, SE) & LD & & & $0.19(0.04)$ & & Age (F) & [6] \\
\hline CF (Gotland, SE) & ID & $\begin{array}{l}3086 \\
\text { (ba) } 4155\end{array}$ & $0.073(0.036)$ & $0.04(0.02)$ & & Clutch size $(\mathbf{F})$ year, $p e_{f}(\mathbf{R})$ & {$[7]$} \\
\hline CG (Matsalu NP, EST) & LD & $\begin{array}{l}\text { (f) } 1916 \\
\text { (m) } 1864 \\
\text { (ba) } 10652\end{array}$ & & $0.15(0.04)$ & $0.05(0.02)$ & & [8] \\
\hline B species (population) & Trait & $N$ & $V_{a}$ & $h_{f}^{2}$ & $h_{m}^{2}$ & Trait values standardized & Study \\
\hline GT (Vlieland, NL) & LD & $\begin{array}{l}\text { (f) } 371 \\
\text { (m) } 378\end{array}$ & & $0.45(0.15)$ & $-0.14(0.12)$ & Year, age & [9] \\
\hline $\begin{array}{l}\text { GT (Hoge Veluwe, } \\
\text { NL) }\end{array}$ & LD & $\begin{array}{l}\text { (f) } 371 \\
\text { (m) } 517\end{array}$ & & $0.18(0.09)$ & $0.06(0.09)$ & Year, age & \\
\hline GT (Liesbos, NL) & LD & $\begin{array}{l}\text { (f) } 129 \\
\text { (m) } 137\end{array}$ & & $-0.08(0.27)$ & $-0.04(0.24)$ & Year, age & \\
\hline $\begin{array}{l}\text { GT (Oosterhout, } \\
\text { NL) }\end{array}$ & LD & $\begin{array}{l}\text { (f) } 56 \\
\text { (m) } 47\end{array}$ & & $0.14(0.36)$ & $0.06(0.25)$ & Year, age & \\
\hline GT (Wytham, UK) & LD & $\begin{array}{l}\text { (f) } 1332 \\
\text { (m) } 1090\end{array}$ & & $\begin{array}{l}0.24(0.06) / \\
0.16(0.07)^{(*)}\end{array}$ & $0.21(0.06)$ & Year, age & [10] \\
\hline BT (Revinge, SE) & LD & (f) 40 & & $0.44(0.38)$ & & Year & [11] \\
\hline CF (Gotland, SE) & LD & (f) 1599 & 14 & $0.41(0.08)$ & & Age & [12] \\
\hline CF (Gotland, SE) & LD & (f) 248 & & $0.29(0.12)$ & & & [13] \\
\hline $\begin{array}{l}\text { TS (Creston Valley, } \\
\text { CAN) }\end{array}$ & LD & (f) 9 & & $1.44(0.52)$ & & & [14] \\
\hline $\begin{array}{l}\text { SS (Mandarte Island, } \\
\text { CAN) }\end{array}$ & LD & (f) 71 & & -0.012 & & Year & [15] \\
\hline C (Aalsmee, NL) & LD & (f) 125 & & $0.02(0.13)$ & & Age, year & [16] \\
\hline $\begin{array}{l}\text { SH (South Scotland, } \\
\text { UK) }\end{array}$ & LD & (f) 53 & & 0.08 & & & [17] \\
\hline $\begin{array}{l}\text { LSG (La Perouse } \\
\text { Bay, CAN) }\end{array}$ & $\mathrm{OH}$ & (f) 136 & & $0.44(0.16)$ & & Year (std) & {$[18]$} \\
\hline PJ (Foula, Shetland) & $\mathrm{OH}$ & $\begin{array}{l}\text { (f) } 10 \\
\text { (m) } 16\end{array}$ & & $-0.2(0.5)$ & $0.7(0.72)$ & Year (std) & [19] \\
\hline
\end{tabular}

Estimates in boldface denote significance. Values listed for $n$ refer to the number of females $(f)$, males $(m)$ and breeding attempts ( $b a)$; fixed and random effects included in the models are indicated by $(\mathbf{F})$ and $(\mathbf{R})$, respectively. Estimates indicated by ${ }^{(*)}$ are corrected for spatial autocorrelation. BT = Blue tit Cyanistes caeruleus, $\mathrm{C}=$ Coot Fulica atra, $\mathrm{CF}=$ Collared flycatcher Ficedula albicollis, $\mathrm{CG}=$ Common gull Larus canus, GT $=$ Great tit Parus major, PJ = Parasitic Jaeger Stercorarius parasiticus, LSG = Lesser snow goose Anser caerulescens c., $\mathrm{SH}=$ Sparrowhawk Accipiter nisus, SS = Song sparrow Melospiza melodia, TS = Tree swallow Tachycineta bicolor.

References: [1] this study, [2] Caro et al., 2009; [3] Gienapp et al., 2006; [4] Garant et al., 2008; [5] McCleery et al., 2004; [6] Sheldon et al., 2003; [7] Husby et al., 2012; [8] Brommer \& Rattiste, 2008; [9] van Noordwijk et al., 1981; [10] van der Jeugd \& McCleery, 2002; [11] Svensson, 1997; [12] Merilä \& Sheldon, 2000; [13] Gustafsson, 1986; [14] Wiggins, 1991; [15] Hochachka, 1990; [16] Perdeck \& Cave, 1992; [17] Newton \& Marquiss, 1984; [18] Findlay \& Cooke, 1982; [19] Phillips \& Furness, 1998. 
environment effects. At the same time it is important to realize that very low dispersal leads to relatives living in the same area, and thus, aspects of the environment could become confounded with genetic variation. The importance of controlling for nest box and permanent environment effects is likely to be species specific and may depend upon species life history (also see McCleery et al., 2004). As our study demonstrates, environmental effects (as measured by nest box and permanent environment effects) can even differ in magnitude between closely related species inhabiting the same woods.

With the caveat that the amount of genetic variation in reproductive timing in the study populations was lower than expected, the results generally suggest that estimates of additive genetic variation were not influenced by the variation in the Clock gene. The timing of breeding is likely to be controlled by a complex network of morphological, behavioural and physiological adaptations, which are probably controlled by a whole suite of genes (recently reviewed by Visser et al., 2010). Our analyses concentrate on Clock, as this is currently the only candidate gene characterized in a context on the timing of reproduction in birds. However, we want to stress that complex life-history traits are generally environmentally influenced, and typically polygenic, and timing of breeding is most likely influenced by the effects of genetic variants of a number of genes involved in a complex network, which are difficult to identify in wild populations (see recent review by Visser et al., 2010). With traits that are highly polygenic, it may therefore not be surprising that candidate genes account for a very small amount of additive genetic variation. However, given that Johnston et al. (2011) found that 76\% of additive genetic variation was explained by a QTL, it remains an open question whether additive genetic variation in wild populations arises through many genes with small effects or a few genes with large effects. Although the answer probably lies in both, it will be important to reveal what type of traits are influenced by many small effect genes and which are affected by few genes with large effects.

In summary, our study demonstrates that there is a little genetic variation in timing parameters, which is much lower than generally assumed, at least in blue tits and great tits. The rather small but targeted effect in female blue tits that was picked up using a candidate gene approach was not detected in a quantitative genetic analysis of the same data. These results suggest that the two approaches do not measure overlapping sources of genetic variation. Importantly however, the degree of additive genetic variation detected in our analyses and the candidate gene effects of Clock are small and thus strongly limit our ability to assess whether candidate gene and quantitative genetic analyses measure similar genetic variation in traits. Our results highlight that quantitative genetic analyses and candidate gene tech- niques may be useful for measuring different genetic effects in wild populations, but this remains to be verified using traits exhibiting higher levels of genetic variation. Given the rapid development of methodological and molecular techniques, we expect combining quantitative and molecular genetic analyses will become a useful approach to studying evolution in wild populations, but which traits this will be successful for remains to be established.

\section{Acknowledgments}

We are grateful to the very large number of fieldworkers that collected the data analysed here, work supported by a range of funding sources including NERC, BBSRC and the Royal Society, and to Ian White for providing R code to calculate standard errors on heritabilities. This work was supported by a Marie Curie Intra-European Fellowship (MEIF-CT-2006-040639) and a Feodor Lynen Fellowship (Alexander von Humboldt-Foundation) to ML and by the Browne Research Fellowship from The Queen's College Oxford to CKC.

\section{References}

Brommer, J.E. \& Rattiste, K. 2008. "Hidden" reproductive conflict between mates in a wild bird population. Evolution 62: 2326-2333.

Caro, S.P., Charmantier, A., Lambrechts, M.M., Blondel, J., Balthazart, J. \& Williams, T.D. 2009. Local adaptation of timing of reproduction: females are in the driver's seat. Funct. Ecol. 23: 172-179.

Charmantier, A., Perrins, C.M., McCleery, R. \& Sheldon, B.C. 2006. Age-dependent variance in a life-history trait in the mute swan. Proc R Soc B., 273: 225-232.

Charmantier, A., McCleery, R.H., Cole, L.R., Perrins, C.M., Kruuk, L.E.B. \& Sheldon, B.C. 2008. Adaptive phenotypic plasticity in response to climate change in a wild bird population. Science 320: 800-803.

Cresswell, W. \& McCleery, R.H. 2003. How great tits maintain synchronization of their hatch date with food supply in response to long-term variability in temperature. J. Anim. Ecol. 72: 356-366.

Ellegren, H. \& Sheldon, B.C. 2008. Genetic basis of fitness differences in natural populations. Nature 452: 169-175.

Findlay, C.S. \& Cooke, F. 1982. Breeding synchrony in the Lesser Snow Goose (Anser caerulescens caerulescens). I. Genetic and environmental components of hatch date variability and their effects on hatch synchrony. Evolution 36: $342-351$.

Fitzpatrick, M.J., Ben-Shahar, Y., Smid, H.M., Vet, L.E.M., Robinson, G.E. \& Sokolowski, M.B. 2005. Candidate genes for behavioural ecology. Trends Ecol. Evol. 20: 96-104.

Garant, D., Kruuk, L.E.B., McCleery, R.H. \& Sheldon, B.C. 2004. Evolution in a changing environment: a case study with great tit fledging mass. Am. Nat. 154: E115-E129.

Garant, D., Hadfield, J.D., Kruuk, L.E.B. \& Sheldon, B.C. 2008. Stability of genetic variance and covariance for reproductive characters in the face of climate change in a wild bird population. Mol. Ecol. 17: 179-188. 
Gienapp, P., Postma, E. \& Visser, M.E. 2006. Why breeding time has not responded to selection for earlier breeding in a songbird population. Evolution 60: 2381-2388.

Gilmour, A.R., Gogel, B.J., Cullis, B.R. \& Thompson, R.. 2009. ASReml user guide release 3.0. VSN International Ltd, Hemel Hempstead, HPI IES, UK.

Grieco, F., van Noordwijk, A.J. \& Visser, M.E. 2002. Evidence for the effect of learning on timing of reproduction in blue tits. Science 296: 136-138.

Gustafsson, L. 1986. Lifetime reproductive success and heritability: Empirical support for Fisher's fundamental theorem. Am. Nat. 128: 761-764.

Hochachka, W. 1990. Seasonal decline in reproductive performance of song sparrows. Ecology 71: 1279-1288.

Hoekstra, H.E. \& Coyne, J.A. 2007. The locus of evolution: evo devo and the genetics of adaptation. Evolution 61: 995-1016.

Husby, A., Gustafsson, L. \& Qvarnström, A. 2012. Low genetic variance in the duration of the incubation period in a collared flycatcher (Ficedula albicollis) population. Am. Nat. 179: 132136.

van der Jeugd, H.P. \& McCleery, R. 2002. Effects of spatial autocorrelation, natal philopatry and phenotypic plasticity on the heritability of laying date 832. J. Evol. Biol. 15: 380-387.

Johnsen, A., Fidler, A.E., Kuhn, S., Carter, K.L., Hoffmann, A., Barr, I.R. et al. 2007. Avian Clock gene polymorphism: evidence for a latitudinal cline in allele frequencies. Mol. Ecol. 16: $4867-4880$

Johnston, S.E., McEwan, J.C., Pickering, N.K., Kijas, J.W., Beraldi, D., Pilkington, J.G. et al. 2011. Genome-wide association mapping identifies the genetic basis of discrete and quantitative variation in sexual weaponry in a wild sheep population. Mol. Ecol. 20: 2555-2566. doi: 10.1111/j.1365294X.2011.05076.X

Kruuk, L.E.B., Slate, J. \& Wilson, A.J. 2008. New answers for old questions: the evolutionary quantitative genetics of wild animal populations. Annu. Rev. Ecol. Evol. Syst. 39: 525-548.

Liedvogel, M. \& Sheldon, B.C. 2010. Low variability and absence of phenotypic correlates of Clock gene variation in a great tit Parus major population. J. Avian Biol. 41: 543-550.

Liedvogel, M., Szulkin, M., Knowles, S.C.L., Wood, M.J. \& Sheldon, B.C. 2009. Phenotypic correlates of Clock gene variation in a wild blue tit population: evidence for a role in seasonal timing of reproduction. Mol. Ecol. 18: 2444-2456.

Lynch, M. \& Walsh, J.B. 1998. Genetics and Analysis of Quantitative Traits. pp. 980. Sinauer Assocs., Inc., Sunderland, MA.

Matthysen, E., van de Casteele, T. \& Adriaensen, F. 2005. Do sibling tits (Parus major, P. caeruleus) disperse over similar distances and in similar directions? Oecologia 143: 301-307.

McCleery, R.H., Pettifor, R.A., Armbruster, P., Meyer, K. $\delta$ Sheldon, B.C. 2004. Components of variance underlying fitness in a natural population of the great tit Parus major. Am. Nat. 164: E62-E72.

Merilä, J. \& Sheldon, B.C. 2000. Lifetime reproductive success and heritability in nature. Am. Nat. 155: 301-310.

Minot, E.O. \& Perrins, C.M. 1986. Interspecific interference competition-nest sites for blue and great tits. J. Anim. Ecol. 55: 331-350.

Morrissey, M.B. \& Wilson, A.J. 2010. pedantics, an R package for pedigree-based genetic simulation and pedigree manipulation, characterization and viewing. Mol. Ecol. Resources. 10: 711-719.

Newton, I. \& Marquiss, M. 1984. Seasonal trend in the breeding performance of sparrowhawks. J. Anim. Ecol. 53: 809-829. van Noordwijk, A.J., Vanbalen, J.H. \& Scharloo, W. 1981. Genetic-variation in the timing of reproduction in the Great tit. Oecologia 49: 158-166.

Nussey, D.H., Postma, E., Gienapp, P. \& Visser, M.E. 2005. Selection on heritable phenotypic plasticity in a wild bird population. Science 310: 304-306.

Perdeck, A.C. \& Cave, A.J. 1992. Laying date in the Coot: effects of age and mate choice. J. Anim. Ecol. 61: 13-19.

Perrins, C.M. 1965. Population fluctuations and clutch-size in the Great tit Parus major L. J. Anim. Ecol. 34: 601-647.

Phillips, R.A. \& Furness, R.W. 1998. Measurement of heritability of hatching date and chick condition in parasitic jaegers. Can. J. Zool. 76: 2290-2294.

Piertney, S.B. \& Webster, L.M.I. 2010. Characterising functionally important and ecologically meaningful genetic diversity using a candidate gene approach. Genetica 138: 419-432.

Self, S.G. \& Liang, K.Y. 1987. Large sample properties of the maximum likelihood estimator and the likelihood ratio test on the boundary of the parameter space. J. Am. Stat. Assoc. 82: 605-611

Sheldon, B.C., Kruuk, L.E.B. \& Merila, J. 2003. Natural selection and inheritance of breeding time and clutch size in the collared flycatcher. Evolution 57: 406-420.

Slate, J., Santure, A.W., Feulner, P.G.D., Brown, E.A., Ball, A.D., Johnston, S.E. et al. 2010. Genome mapping in intensively studied wild vertebrate populations. Trends Genet. 26: 275-284.

Svensson, E. 1997. Natural Selection on Avian Breeding Time: causality, Fecundity-Dependent, and Fecundity-Independent Selection. Evolution 51: 1276-1283.

Tabor, H.K., Risch, N.J. \& Myers, R.M. 2002. Candidate-gene approaches for studying complex genetic traits: practical considerations. Nat. Rev. Genet. 3: 391-397.

Tauber, E. \& Kyriacou, C.P. 2005. Molecular evolution and population genetics of circadian clock genes. Circadian Rhythms. 393: 797-817.

Thomas, D.W., Blondel, J., Perret, P., Lambrechts, M.M. \& Speakman, J.R. 2001. Energetic and fitness costs of mismatching resource supply and demand in seasonally breeding birds. Science 291: 2598-2600.

Venables, W.N. \& Ripley, B.D. 2000. S Programming. SpringerVerlag, New York.

Visser, M.E. 2005. Shifts in phenology due to global climate change: the need for a yardstick. Proc $R$ Soc B. 272: 2561-2569.

Visser, M.E., Caro, S.P., van Oers, K., Schaper, S.V. \& Helm, B. 2010. Phenology, seasonal timing and circannual rhythms: towards a unified framework. Phil. Trans. R. Soc. Lond. B 365 3113-3127.

Visser, M.E., Schaper, S.V., Holleman, L.J.M., Dawson, A., Sharp, P., Gienapp, P. et al. 2011. Genetic variation in cue sensitivity involved in avian timing of reproduction. Funct. Ecol. 25: 868-877.

Wiggins, D.A. 1991. Natural selection on body size and laying date in the tree swallow. Evolution 45: 1169-1174.

Wilkin, T.A., Garant, D., Gosler, A.G. \& Sheldon, B.C. 2006. Density effects on life-history traits in a wild population of the great tit Parus major: analyses of long-term data with GIS techniques. J. Anim. Ecol. 75: 604-615.

Wilson, A.J. 2008. Why $h^{2}$ does not always equal $V_{A} / V_{P}$ ? J. Evol. Biol. 21: 647-650.

Wilson, A.J., Réale, D., Clements, M.N., Morrissey, M.M., Postma, E., Walling, C.A. et al. 2010. An ecologist's guide to the animal model. J. Anim. Ecol. 79: 13-26. 


\section{Supporting information}

Additional Supporting Information may be found in the online version of this article:

Figure $\mathbf{S} \mathbf{1}$ The blue tit pedigree.

Figure S2 The great tit pedigree.

Table S1 The effect of Clock $Q$ genotype on timing of laying (LD).

Table S2 Additive genetic variance in timing of laying (LD).

Table S3 The effect of Clock $Q$ genotype and additive genetic variance in timing of laying (LD).

Table S4 The effect of Clock $Q$ genotype on incubation duration (ID).

Table S5 Additive genetic variance in incubation duration (ID).

Table S6 The effect of Clock $Q$ genotype and additive genetic variance in incubation duration (ID).

Table S7 The effect of Clock $Q$ genotype on observed hatch date $(\mathrm{OH})$.

Table S8 Additive genetic variance in observed hatch date $(\mathrm{OH})$.

Table S9 The effect of Clock $Q$ genotype and additive genetic variance in observed hatch date $(\mathrm{OH})$.

Table S10 Additive genetic variance in timing of laying (LD) calculated using full dataset.

Table S11 Additive genetic variance in incubation duration (ID) calculated using full dataset.

Table S12 Additive genetic variance in hatch date $(\mathrm{OH})$ calculated using full dataset.

Table S13 Additive genetic variance in fledging mass calculated using clock dataset and restricted to same number of datapoints.

Table S14 Additive genetic variance in fledging mass calculated using full dataset.

Table S15 Additive genetic variance in timing of laying (LD).

Table S16 The effect of Clock $Q$ genotype and additive genetic variance in timing of laying (LD).

Table S17 The effect of Clock $Q$ genotype on incubation duration (ID).

Table S18 Additive genetic variance in incubation duration (ID).

Table S19 The effect of Clock $Q$ genotype and additive genetic variance in incubation duration (ID).

Table S20 Additive genetic variance in observed hatch date $(\mathrm{OH})$.

Table S21 The effect of Clock $Q$ genotype and additive genetic variance in observed hatch date $(\mathrm{OH})$.

Table S22 Additive genetic variance in timing of laying (LD) calculated using full dataset.

Table S23 Additive genetic variance in incubation duration (ID) calculated using full dataset.

Table S24 Additive genetic variance in hatch date $(\mathrm{OH})$ calculated using full dataset.
Table S25 Additive genetic variance in fledging mass calculated using clock dataset and restricted to same number of datapoints.

Table S26 Additive genetic variance in fledging mass calculated using full dataset.

Table S27 The effect of additive genetic variance and Clock $Q$ genotype fitted as random effect on lay date in blue tits.

Table S28 The effect of additive genetic variance and Clock $Q$ genotype fitted as random effect on incubation duration in blue tits.

Table S29 The effect of additive genetic variance and Clock $Q$ genotype fitted as random effect on hatch date in blue tits.

Table S30 The effect of additive genetic variance and Clock Q genotype fitted as random effect on lay date in female great tits.

Table S31 The effect of additive genetic variance and Clock $Q$ genotype fitted as random effect on incubation duration in female great tits.

Table S32 The effect of additive genetic variance and Clock $Q$ genotype fitted as random effect on hatch date in female great tits.

Table S33 Estimates of additive genetic variance for lay date in blue tits using the full phenotypic dataset without including fixed and random factors.

Table S34 Estimates of additive genetic variance for incubation duration in blue tits using the full phenotypic dataset without including fixed and random factors.

Table S35 Estimates of additive genetic variance for observed hatch date in blue tits using the full phenotypic dataset without including fixed and random factors.

Table S36 Estimates of additive genetic variance for lay date in great tits using the full phenotypic dataset without including fixed and random factors.

Table S37 Estimates of additive genetic variance for incubation duration in great tits using the full phenotypic dataset without including fixed and random factors.

Table S38 Estimates of additive genetic variance for observed hatch date in great tits using the full phenotypic dataset without including fixed and random factors.

As a service to our authors and readers, this journal provides supporting information supplied by the authors. Such materials are peer-reviewed and may be reorganized for online delivery, but are not copy-edited or typeset. Technical support issues arising from supporting information (other than missing files) should be addressed to the authors.

Data deposited at Dryad: doi: 10.5061/dryad.6n3d6109

Received 20 October 2011; revised 30 January 2012; accepted 30 January 2012 